\title{
Method for experimental data processing in the sphere of hydrodynamics of packed heat and mass exchange apparatuses
}

\author{
Nikolay Merentsov ${ }^{1}$,Vyacheslav Balashov ${ }^{1}$, Denis Bunin $^{2}$, Vitaliy Lebedev ${ }^{2}$, Aleksandr Persidskiy $^{3}$, \\ and Mikhail Topilin ${ }^{2}$ \\ ${ }^{1}$ Volgograd State Technical University, 400005 Volgograd, Russia \\ ${ }^{2}$ Branch of LUKOIL-Engineering VolgogradNIPImorneft, 400078 Volgograd, Russia \\ ${ }^{3}$ JSC Federal Scientific and Production Centre «Titan - Barricady», 400071 Volgograd, Russia
}

\begin{abstract}
The article dwells on the method for summarizing experimental data on hydrodynamics of one-phase filtration flows through packed heat-and-mass exchange apparatuses differing in configuration. Summarizing is performed using a modified generalized equation. The described method simplifies the work with experimental bulk data, helps to compare the packed heat-andmass exchange apparatuses, assess their energy performance and find the ways for their industrial implementation in heat-and mass exchange equipment.
\end{abstract}

\section{Introduction}

A large quantity of industrial packingsis known and a lot of packings are offered to be used industrially [1-13], and the work aimed at the creation of new packings is still going on. Creation of new highly efficient packings is a difficult and time-consuming process that sometimes requires providing for necessary but colliding structural solutions [1-19].

Requirements set for the packings of industrial apparatuses are low flow resistance, a wide range of liquid and gas load, ability to form a uniform velocity profile in the cross-section of the apparatus, good wettability of the packing (and in some cases hydrophobic behaviour, when it is necessary to reach dripping water flow mode), high retaining capacity for liquid, resistance to aggressive media, sufficient mechanical strength and low cost. The variety of packings used industrially is reasoned by the necessity to ensure high efficiency of the process equipment operation.

One of the key performance indices for a heat-andmass exchange apparatus without regard to the details of the process is its resistance without wetting. A heat-andmass exchange apparatus working without wetting and penetrated only by a flow of gas (air) is called "dry". Flow resistance of dry packed-type device is the parameter that is used first of all to assess its industrial implementation $[14,15]$.

\section{Experimental part}

The relation between flow resistance of a dry packed bed having certain height and gas (air) filtration rate divided by the total cross-section of the packed bed is called a filtration curve. Irrespectively of the type, model and construction of the packed-type device filtration curve $\Delta p / H=f\left(v_{f}\right)$ is a monotonous curve starting from the origin of coordinates [14,15]. Figure 1 shows filtration curves in one coordinate space $\Delta p / H=f\left(v_{f}\right)$. The position of the filtration curves in the coordinate space is governed by permeability of the inner structure of the packed bed. Flow resistance of the exchanger is in inverse relation to its permeability, thus, filtration curve 1 corresponds to the bed with the highest permeability, and curve 2 - to the bed with the lowest permeability.

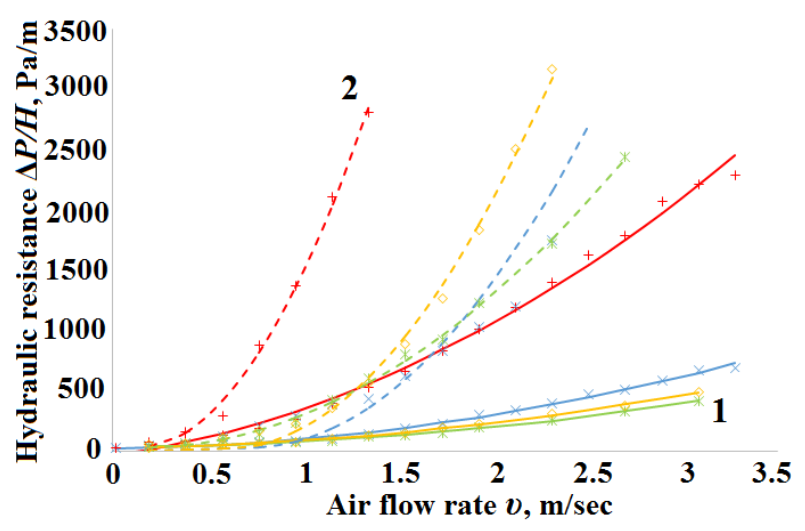

Fig. 1. Filtration curves of $\Delta p / H=f\left(v_{f}\right)$. 
The photo and the scheme of the experimental modular plant can be considered in Figures 2 and 3.

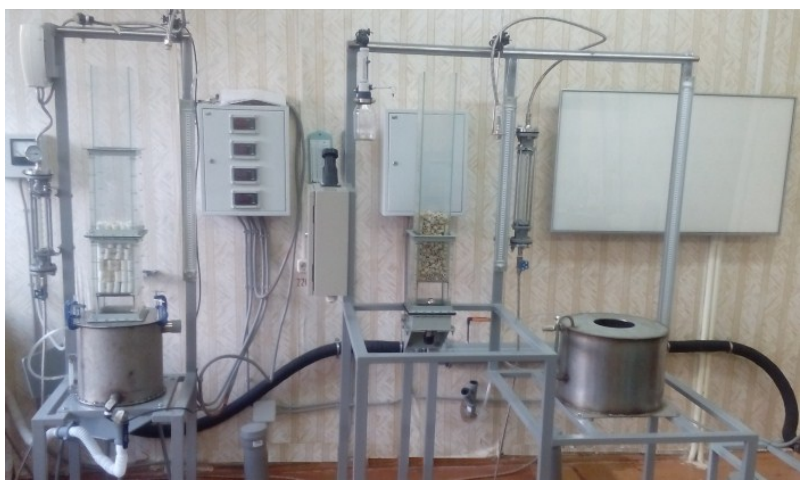

Fig. 2. Experimental modular plant for studying hydrodynamics and heat-and-mass exchange inpacked-type devices.

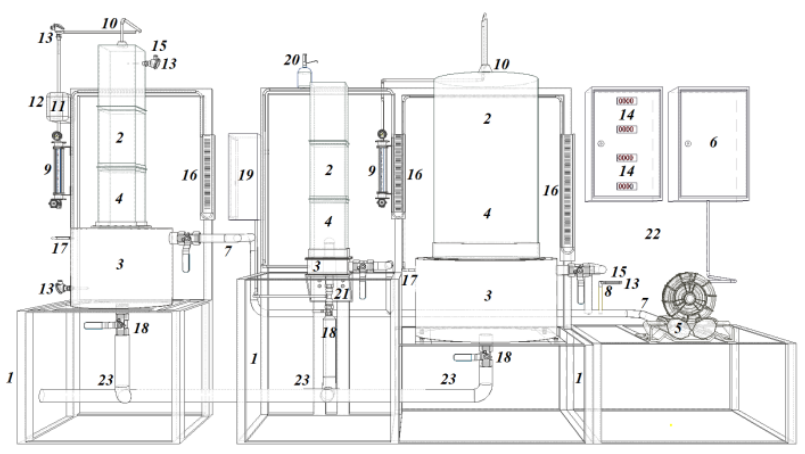

Fig.3. The scheme of the experimental modular plant: 1 - a supporting frame; 2 - a column body; 3 - a catchment and gas-distributing sampler; 4 - investigated packed devices in cartridges; 5 - a pressure blower; 6 - frequency converters; 7 - an air-conditioning duct; 8 - a gas flow meter indicating the speed and a volumetric flow rate; 9 - variable area flow meters; 10 - replaceable liquid distributors; 11 - an instantaneous water heater; 12 - a potentiometer of adjusting the heating of water; 13 - temperature sensors; 14 microprocessor devices processing the signal of temperature sensors; 15 - moisture content sensors; 16 - differential pressure gages; 17 - liquid level indicators in the samplers; 18 - quick-detachable drain valves (necessary for experimental research of the holding capacity); 19 - a block for reading of response curves for the flow structures interpretation; $20-\mathrm{a}$ mechanical measure feeder of indicator solutions; 21 changeable electrode groups; 22 - a screen for applying calibration charts (presented on the photo); 23 - a pipe for draining water into the sewer.

\section{Methods and calculations}

Filtration curve in the wide range of filtration rate values is described by Dupuit-Forchheimer equation

$$
\frac{\Delta p}{H}=\alpha \mu v_{f}+\beta \rho v_{f}^{2},
$$

where $\Delta p$ is flow resistance of the packed bed, $H$ is height of the packed bed, $v_{f}$ is filtration rate divided by the total cross-section of the packed bed, $\mu$ and $\rho$ are viscosity and density of gas (air), $\alpha$ and $\beta$ are viscous and inertial resistance factors.
There are always viscous friction and inertial forces in a filtration flow. Viscous and inertial resistance factors are the characteristics of the packed-type device that show the influence of its inner structure on the formation of viscous friction and inertial forces in the filtration flow. In the area of small filtration rates viscous friction forces greatly exceed inertial forces which can even be neglected. In the area of high filtration rates inertial forces contrarily prevail, and viscous friction forces become small to negligible compared to them. Taking the above described assumptions into account the second member can be omitted in equation (1) in the area of small rates, and the first member can be omitted in the area of high rates. Thus, for filtration flow of gas (air) in the area of small filtration rates flow resistance of the packed bed can be calculated as $\Delta p / H=\alpha \cdot \mu \cdot v_{f}$; such filtration mode is called laminar. For the area of high rates flow resistance of the packedbed can be calculated as $\Delta p / H=\beta \cdot \rho \cdot v_{f}^{2}$; such filtration mode is called turbulent. The monotonous character of the filtration curve is reasoned by the steady increase of inertial forces in the flow of gas (air) in addition to viscous friction forces and gradual change of laminar filtration mode by developed turbulent mode without any abrupt changes. It is evident that with such dynamics of viscous friction forces and inertial forces at the filtration curve there is a transition filtration zone with a steadily increasing exponential of turbulization between the areas of laminar filtration and developed turbulent filtration. It is also evident that the boundaries of these filtration modes can be determined only conventionally depending on the scale of graphic presentation of the filtration curve and required accuracy of calculation.

Flow resistance of a dry packed bed is calculated by equation

$$
\Delta p_{d r y}=\lambda \frac{H}{l_{x}} \frac{v_{f}^{2} \rho}{2 \varepsilon^{2}},
$$

where $\lambda$ is packed bed resistance factor; $l_{x}$ is reference linear dimension of the packed bed geometrical structure. The value of resistance factor is determined using generalized relation $\lambda=f\left(R e_{g}\right)$, where

$$
\operatorname{Re}_{g}=\frac{v_{f} \rho l_{x}}{\mu \varepsilon},
$$

is Reynolds number for filtration flow of gas (air).

Generalized relation $\lambda=f\left(R e_{g}\right)$ is determined by experiment. Empirical equation for its approximation is picked only within the range of Reynolds number values measured in the experiments. If the experiments, for example, were performed in the area of a small range of Reynolds number values on the transition area between laminar and developed turbulent filtration mode, then an exponential equation can be used as an approximating function. If the range of the experimental study includes areas of laminar and partly transition modes of filtration flow, a linear-exponential equation can be used for a certain interval as an approximating function. The necessity to receive a generalized relation for a narrow range using Reynolds number is observed when it is necessary to determine flow resistance of the packedtype device operating within a known range of rates of 
gas (air). This flow resistance determines threshold values of Reynolds number for the experiments.

When performing an experiment within a wide range of Reynolds number (Modified $R e_{g}$ range 0,001-100) values including the areas of all filtration modes of gas (air) flow, the following approximating function is used

$$
\lambda=\frac{A}{\operatorname{Re}_{g}}+B,
$$

where $A, B$ are constant factors. It is necessary to receive generalized relation $\lambda=f\left(R e_{g}\right)$ that summarizes experimental data fora wide range of modes when performing research and development works aimed at the determination of the possible working range of the studied packed device type with respect to its gas (air) rate and at the assessment of its efficiency compared to the known types of packed devices used in industry.

Values of $A$ and $B$ are determined by experiment. For that curve equation (4) is linearized as function

$$
\lambda \cdot \operatorname{Re}_{g}=A+B \cdot \operatorname{Re}_{g},
$$

which in new variables $\lambda \cdot \mathrm{Re}_{g}=f\left(\mathrm{Re}_{g}\right)$ is a straightline equation. Using the data of the experiments aimed at the determination of flow resistance of dry packed bed $\Delta p / H=f\left(v_{f}\right)$ and accepting the packed bed equivalent diameter as reference length $l_{\text {ref }}=d_{e q}=4 \varepsilon / \sigma$, we calculate Reynolds number values

$$
\operatorname{Re}_{g}=\frac{v_{f} \rho l_{x}}{\mu \varepsilon}
$$

and values of $\lambda \cdot R e_{g}$ complex. Value of $\lambda$ factor is calculated using equation

$$
\lambda=\frac{\Delta p_{d r y}}{H} \cdot \frac{2 \cdot l_{x} \cdot \varepsilon^{2}}{v_{f}{ }^{2} \cdot \rho},
$$

received from formula (2). For calculated values of the complex and Reynolds number dots are marked in the coordinate space $\lambda \cdot R e_{g}-R e_{g}$. These dots are used to build the line that approximates the required relation. The tangent of an angle between the line and the $R e_{g}$ axis and the interval that it cuts by the $\lambda \cdot R_{g}$ axis are used to calculate $A$ and $B$ factors.

$A$ and $B$ factors can be calculated by another method. It is necessary to substitute in equation (5) the coordinates taken from the line diagram for the two end points and receive a set of two algebraic equations which can be used to calculate the values of $A$ and $B$ factors.

The generalized equation that can be used to determine resistance factor for a group of packed devices of another type is called summarizing. Values of $A$ and $B$ factors for the summarizing equation can be determined as follows. Experiments are carried out for the studied group of packed-type devices aimed at the determination of their flow resistance $\Delta p / H=f\left(v_{f}\right)$.

Following the method described above dots are marked in the coordinate space $\lambda \cdot R e_{g}-R e_{g}$ for each type of packed devices. The received dots are approximated by one line which is used to calculate $A$ and $B$ factors for generalized equation (5) which with such values of $A$ and $B$ factors will be generalized for the studied group of packed devices. Actual resistance of the packed bed can differ from the value calculated using the summarizing generalized equation. The expected difference is assessed during mathematical treatment of the experimental data using correlation and regression analyses.

The use in the equation for determination of flow resistance of the packed bed (2) and expression of Reynolds number (3) as reference linear dimension of equivalent diameter, $l_{r e f}=d_{e q}=4 \varepsilon / \sigma$, allows for summarizing of filtration experimental data in the form of generalized equation (6) only for some groups of regular and irregular packed-type devices having similar geometrical form of elements forming the inner structure of the packed bed that produces a large quantity of equations for determination of flow resistance factor. A large quantity of such generalized equations, variety of their publications and often absence of information on the boundaries of their application and the possibility that there exist other limits makes the comparison of the efficiency of work of exchangers of different types complex very difficult.

Theoretical and experimental studies show that use of not one, but two reference linear dimensions as hydraulic characteristics of the packed-type device determined by viscous and inertial resistance factors in the DupuitForchheimer equation (1) as

$$
l_{x 1}=\frac{1}{\beta}
$$

and:

$$
l_{x 2}=\frac{\beta}{\alpha}
$$

allows to obtain one generalized equation to summarize the experimental data for filtration on all known types of packed-type devices. Values of $\alpha$ and $\beta$ factors required for determination of $l_{x 1}$ and $l_{x 2}$ are determined experimentally. Reference linear dimension $l_{x l}$ is the parameter of the packed-type device that takes into account the value of inertial component of fluid resistance, and $l_{x 2}$ is the parameter of the packed-type device that takes into account the combined action of viscous friction and inertial forces. Value of $l_{x l}$ is plugged into equation (6) instead of $l_{x} \cdot 2 \varepsilon^{2}$ complex, and it can be written in a new format as

$$
\Delta p_{d r y}=\lambda^{*} \frac{H}{l_{x 1}} v_{f}^{2} \rho=\lambda^{*} H \beta v_{f}^{2} \rho .
$$

Value of $l_{x 2}$ is plugged into the expression of Reynolds number (3) which in a new format looks as

$$
\operatorname{Re}_{g}^{*}=\frac{v_{f} \rho l_{x 2}}{\mu}=\frac{v_{f} \rho \beta}{\alpha \mu} .
$$

In these expressions $\lambda^{*}$ and $R e_{g}{ }^{*}$ are new modified values of resistance factor and Reynolds number. It can be said that when using modified values of resistance factor and Reynolds number the approximating function (9) is transformed into a new modified generalized equation

$$
\lambda^{*}=\frac{2}{\operatorname{Re}_{g}^{*}}+2
$$


whichis general for all known types, models and structures of packed devices. Equations (6) and (9) express mathematically the relation between resistance factor and Reynolds number for the experiments on gas filtration through different packed-type devices. At that for establishing this relation in the form of a modified equation (11) as compared to equation (4) it is not necessary to determine values of $A$ and $B$ factors, because their values are already known and equal one (two), and this demonstrates that modified generalized relation (11) is applicable to summarize experimental data for all types of packed devices [14,15,19-21], the results of processing of which are shown as corresponding ranges at the summarizing curve in Figure 4.

Using this method we can process experimental data for all known and newly created heat-and-mass exchange apparatuses, and each studied apparatus will find its mode range at curve $\lambda=f\left(\operatorname{Re}_{g}\right)$ that allows to compare the energy performance and recommend certain heat-and-mass exchange apparatuses for efficient industrial application.

But one should note that this generalized equation is applicable only when the geometrical structure of the packed-type device is characterised by two linear dimensions, and the values of modified resistance factor $\lambda^{*}$ and modified number $R e_{g}{ }^{*}$ are not equal to the values of resistance factor $\lambda$ and Reynolds number $R e_{g}$, when only one reference linear dimension is used to describe the structure of the packed-type device, it is equivalent diameter $d_{e q}$.

The Dupuit-Forchheimer equation (1) is linearized by plugging into it the values of viscous and inertial factors $\alpha$ and $\beta$, writing it as

$$
\frac{\Delta p}{H v_{f}}=\alpha \mu+\beta \rho v_{f}
$$

The obtained relation is a straight-line equation in coordinates $\Delta p / H v_{f}-v_{f}$. Using the experimental data on the determination of flow resistance of a dry packed-type device $\Delta p / H=f\left(v_{f}\right)$ we can calculate the values of $\Delta p / H v_{f}$ complex, dots are marked in the coordinate space $\Delta p / H v_{f}-v_{f}$, and they are approximated by a line. The tangent of an angle between the line and the $v_{f}$ axis and the interval that it cuts by the $\Delta p / H v_{f}$ axis are used to calculate $\alpha$ and $\beta$ factors and to determine the values of reference linear dimensions $l_{x 1}$ and $l_{x 2}$ following expressions (7) and (8).

\section{Results and discussion}

Thus, we have received a universal generalized relation $\lambda^{*}=f\left(\operatorname{Re}_{g}{ }^{*}\right)$ using which we can study in detail the operation mode of any heat-and-mass exchange apparatuses that have any configuration and provide recommendations on their efficient industrial use. This relation also helps to assess the energy performance of heat-and-mass exchange apparatuses and select the required process equipment for heat-and-mass transfer.

Generalized equation $\lambda^{*}=f\left(\operatorname{Reg}_{g}{ }^{*}\right)$ is especially valuable, because it is simple, convenient and apparent for working with huge experimental bulk data on hydrodynamics of heat-and-mass exchange apparatuses that we received and described in various papers, because this generalized relation covers a very wide range of rates and resistances. It is also true for designation of the exchangers for such processes as extraction, fractionation, absorption, evaporation cooling, inertial gas scrubbing, etc.

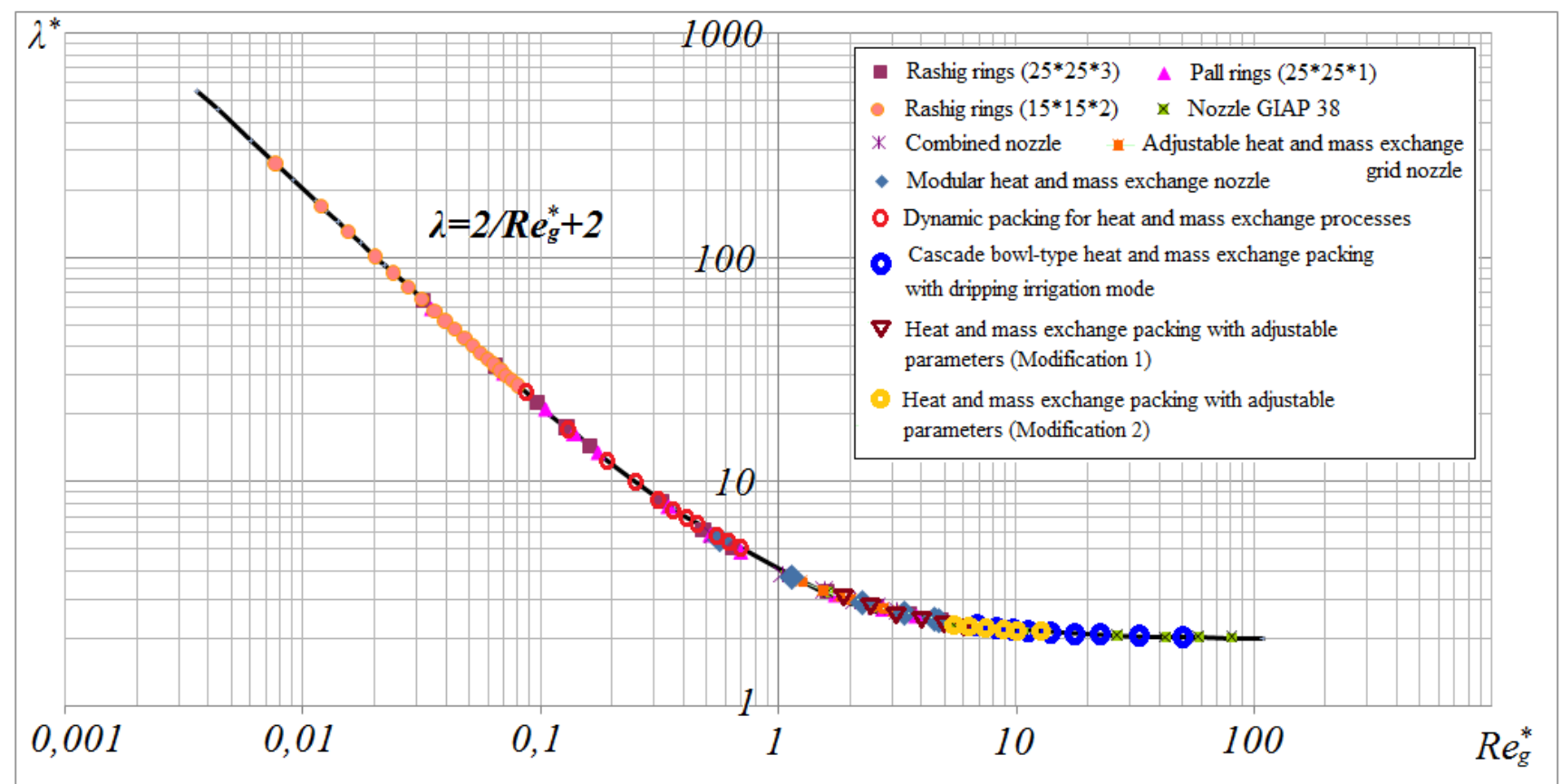

Fig. 4. An example of modified generalized relation $\lambda^{*}=f\left(\operatorname{Re}_{g}{ }^{*}\right)$. 


\section{References}

1. I.N. Madyshev, O.S. Dmitrieva, A.V. Dmitriev, MATEC Web of Conf.,194, 01036 (2018)

2. J. Lee, Nucl. Eng. Des. 326, 65 (2018)

3. C. Zheng, X. Chen, L. Zhu, J. Shi, Energy 150, 653 (2018)

4. A.S.Sokolov, A.S.Pushnov,M.V. Shapovalov, Chem. and Pet. Eng. 53, 1 (2017)

5. A.K.Mitin, N.E. Nikolaikina, A.S.Pushnov, N.A. Zagustina, Chem. and Pet. Eng. 52, 47 (2016)

6. I.N. Madyshev, O.S. Dmitrieva, and A.V. Dmitriev, MATEC Web of Conf. 194, 01013 (2018)

7. Y. Sun, Z. Guan, K. Hooman, Renew. Sust. Energ. Rev. 79, 618 (2017)

8. A.A.Gorodilov, A.S.Pushnov, M.G. Berengarten, Chem. and Pet. Eng. 50, 84 (2014)

9. G.V. Kuznetsov, D.V. Feoktistov, E.G. Orlova, S. Y. Misyura, V.S. Morozov, A.G. Islamova, Int. J. Heat Mass Transfer 126, 161 (2018)

10. O.S. Dmitrieva, A.V. Dmitriev, A.N. Nikolaev, Chem. Pet. Eng. 50, 169 (2014)

11. K. Ponomarev, E. Orlova, D. Feoktistov, EPJ Web Conf. 110, 01060 (2016)

12. V. Maksimov, T. Sharifulin, L. Zhidkova, K. Eloyan, EPJ Web Conf. 159, 00033 (2017)

13. M.Shilyaev, E.Khromova, and A.Bogomolov, MATEC Web of Conf. 115, 08006 (2017)

14. B.A. Sokol, A.K. Chermyshov, D.A. BaranovMasstransfer column packed-type devices (Infokhim, Moscow, 2009)

15. A.M. Kagan, A.G. Laptev, A.S. Pushnov, M. I. Farakhov Contact packings in industrial heatand-mass transfer apparatuses (Otechestvo, Kazan, 2013)

16. S.Shevelev, MATEC Web of Conf. 194, 01053 (2018)

17. Y. Li, X. You, Q. Qiu, J. Li, Energy Convers. Manage. 52, 53 (2011)

18. S.Shevelev, MATEC Web of Conf. 141, 01015 (2017)

19. N.A. Merentsov, V.N. Lebedev, A.B. Golovanchikov, V.A. Balashov, E.E. Nefed'eva, IOP Conf. Ser: Earth and Environmental Science 115 (2018)

20. A.B. Golovanchikov,V.A. Balashov, N.A. Merentsov, Chem. and Pet. Eng. 53, 10 (2017)

21. A.B. Golovanchikov, N.A. Merentsov, V.A. Balashov, Chem. and Pet. Eng. 48, 595 (2013) 